\title{
Study of Electric Field Enhancement Caused by Debris on Laser Optics (Withdrawal Notice)
}

Hu Huang, Kyle Kafka, Stavros Demos

Hu Huang, Kyle Kafka, Stavros Demos, "Study of Electric Field Enhancement Caused by Debris on Laser Optics (Withdrawal Notice)," Proc. SPIE 11514, Laser-induced Damage in Optical Materials 2020, 115141A (18 September 2020); doi: $10.1117 / 12.2571062$

SPIE. Event: SPIE Laser Damage, 2020, Online Only 


\section{Study of Electric Field Enhancement Caused by Debris on Laser Optics (Withdrawal Notice)}

Hu Huang, Kyle Kafka, Stavros Demos

Univ. of Rochester (United States)

Proceedings Volume 11514, Laser-induced Damage in Optical Materials 2020; 115141 A (2020)

https://doi.org/10.1117/12.2571062

Event: SPIE Laser Damage, 2020, Online Only

Online Publication Date: 11 September 2020

Withdrawn from Publication: 18 September 2020

Publisher's Note: This presentation, originally published on 11 September 2020, was withdrawn per author request. 